\title{
A DISTRIBUTED SOFTWARE FOR COLLABORATIVE SALES FORECASTING
}

\author{
Kary FRÄMLING*, Jan HOLMSTRÖM** \\ *ARCADA Polytechnic, Skogsmansgränden 3, 02130 Espoo, FINLAND \\ kary.framling@arcada.fi.http://www.sit.fi $\sim$ kary \\ ${ }^{* *}$ TAI institute, Helsinki University of Technology, Otakaari 1, 02150 Espoo, FINLAND \\ jan.holmstrom@,hut.fi
}

\begin{abstract}
Main advantages of distributed software are that computers connected over a network may access information stored anywhere in the network at any time and get automatically notified about information changes. This paper describes how a distributed software is built for collaborative demand forecasting. Demand forecasts of both providers and clients can be accessed and used for getting a better idea about the marketplace and thereby do more accurate demand forecasting.
\end{abstract}

Keywords: computer-integrated enterprises, co-operation, decision support systems, information systems, data privacy.

\section{INTRODUCTION}

In a competitive and changing business environment, reliable forecasts are needed both for planning longterm business strategies and ensuring that the supply chain operates effectively on a day-to-day basis.

In the consumer goods business, delivery times to the trade are short and the product ranges are wide. Product ranges are wide because a widely used strategy to increase demand is to increase product variety (Quelch and Kenny, 1994). Few producers are able to allocate capacity to produce according to daily consumer demand. A high delivery service is maintained by supplying from stock. To improve the supply chain efficiency producers strive to forecast sales. If the attempt is successful it is possible to reduce safety stock levels while maintaining the service level.

Suppliers, wholesalers and retailers are today increasingly working together to improve supply chain performance and consumer value (ECR Best Practices Operating Committee, 1995). There are obvious advantages from the co-operation in forecasting and supply chain control. A major advantage is that feedback on consumer demand changes can be quickly communicated upstream to suppliers. Similarly, the trade can benefit from being involved in new product launches and promotion planning.
This paper presents a distributed program whose purpose is to facilitate and accelerate co-operation between the members of the supply chain. Section 2 describes how the members of the supply chain connect together, how the distributed program is set up and managed, as well as the technical solutions behind it.

Section 3 describes the currently used method for doing assortment forecasting (Holmström, 1998). It also explains the kind of data that is moved around in the distributed program. Section 4 concerns data security both from the network point of view and from the data accessibility point of view.

\section{DISTRIBUTED SUPPLY CHAIN MANAGEMENT PROGRAM}

A supply chain usually consists of suppliers, wholesalers and retailers. Some of these are just clients to others, others are just providers, while some are both clients and providers to other members of the supply chain (Fig. 1).

From a software point of view, the client and provider roles are more useful than the supplier, wholesaler and retailer roles. Treating them all as potential clients and/or providers makes it possible to model each member of the supply chain in a uniform way. 


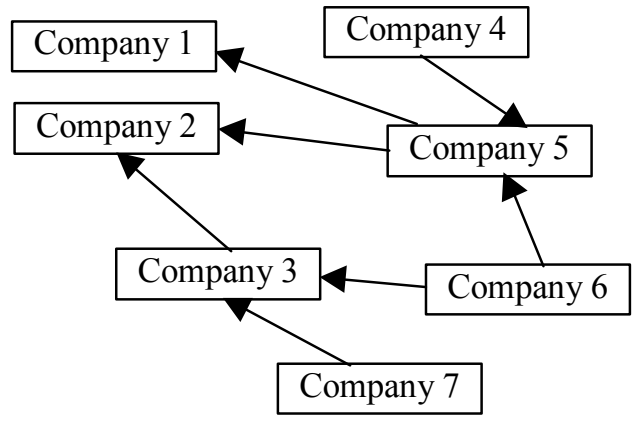

Fig. 1. Supply chain. Arrows indicate product flow, so companies 4,6 and 7 are only providers, companies 1 and 2 are only clients and companies 5 and 3 are both clients and providers.

\subsection{Basic operations}

The two basic operations supported by the system are:

- Updating the company's own forecasts

- Requesting forecast information from the clients and/or providers of the company

The format of forecast information depends on the demand forecasting method used. It is currently implemented for the assortment forecasting method described in Section 3, but the program is designed in a way that makes it possible to easily change the method used.

The intended users of the basic operations are those responsible for category management on the client side, and those responsible for customer relationship on the provider side. The point is that it is the business people, not the logistics people, who are the target users of the system. These are the people who can do the best forecasts and who most need the forecasts of their clients/providers.

\subsection{Administrative operations}

Administrative operations are performed by people who have a sufficient knowledge about the program itself to set it up appropriately. These operations are mainly:

- Adding connections to other members of the supply chain

- Removing connections to other members of the supply chain

- Setting up security issues (described more in detail in Section 4)

The following list is the minimal information needed for each connection:

- Name and description

- Network name/address of the computer that the remote program is running on

- Public key for authentication purposes

\subsection{Technical implementation of the distributed program}

The protocol used is RMI (Remote Method Invocation) (Sun Microsystems, 1998), which is a client-server protocol defined by Sun and which is specific for the Java programming language. Reasons for using the RMI protocol are:

- The program may have to run under different operating systems and over the Internet, so Java is a natural choice.

- Any node of the distributed program may be both a client and a server.

- Easy set-up, because it only requires the presence of a HTTP server and standard Java tools.

- Free, no extra investments needed.

- Easier to understand and control than the Corba protocol (Orfali et al, 1997), for instance. See www.omg.org for information about Corba.

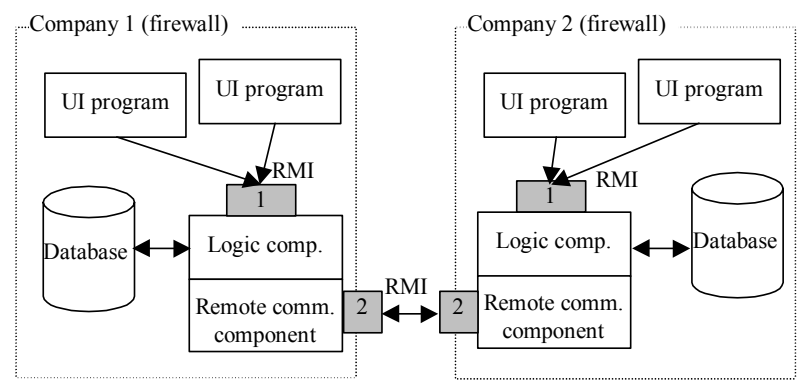

Fig. 2. Main components of the distributed program and the connections between them. The dotted rectangle shows the limits of the firewalls of two different companies.

Fig. 2 shows the main components and the connections between them. There are three main components in the system:

- User interface program for viewing and updating forecasting information

- Remote communication component for managing communication with other companies, including authentication and encryption

- Logic component, which handles data access and updating, as well as connection information to other members of the supply chain

RMI connections are used for communication between these components.

It is obvious why communication between different companies has to be implemented as a remote connection, since the information asking computer and the information providing computer are not the same.

The reason for using RMI in the internal connection between the user interface program and the logic is the same. The user interface program may be started simultaneously by several users on different computers, so it is generally executed on a different computer than the one that contains the data. 


\section{DEMAND FORECASTING}

The demand forecasting method used is Assortment Forecasting (Holmström, 1998). Assortment Forecasting is based on the observation that the cumulative net sales value of products of one assortment tends to be a logarithmic function of the ranking of the product (Fig. 3).

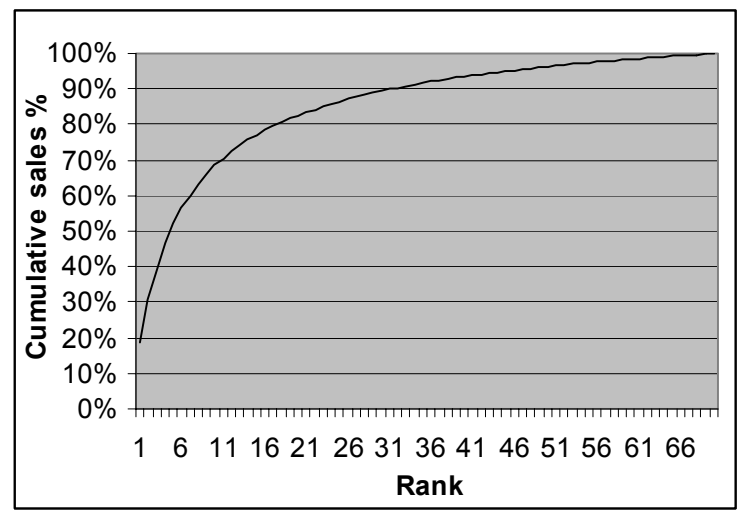

Fig. 3. Example of cumulative percentage of the sales value as a function of rank.

This observation is very interesting because it requires much less work from the sales managers to give a ranking for one product assortment than to forecast the demand for each product based on historical data. Ranking also has the advantage of automatically taking into account the natural competition between products. If one product improves its' sales, then it usually decreases sales of some other products.

The main objective of this method is to offer a simple way of forecasting the effects of change on product sales values. Examples of such changes are:

- launching a new product

- removing an old product

- promotional sales

- products loosing sales value when they have been on the marketplace too long

The current user interface component lets the user visualise a plot like the one in Fig. 3 for any product category. Then the program calibrates a mathematical model that fits as well as possible to the actual plot. The mathematical model used is expressed by equations (1) and (2).

$$
\begin{aligned}
& \text { fraction }(\text { rank })=\frac{1}{(\text { rank }+ \text { const })^{1+\text { power }}} \\
& \text { cumsales } \%(\text { rank })=\sum_{\text {rank }} \frac{\text { fraction }}{\text { total_fraction }}
\end{aligned}
$$

Calibrating is done by changing the values of the const and power parameters until the model fits the actual data as well as possible. The user can vary these parameters in order to simulate the effect of changes in demand, like selling more high-ranking products than usual, for instance.
Then the sales value of each product can be estimated with equation (3). If the total sales value remains constant, then total_sales is already known. If the total sales value is expected to change, then the user can simulate the effects of various total sales values on product sales values.

$$
\text { value }(\text { rank })=\frac{\text { total_sales } * \text { fraction }(\text { rank })}{\text { total_fraction }}
$$

For forecasting purposes, the user can change the ranking by changing the position of products in his ranking. He can also introduce new products or remove existing ones.

\section{SECURITY}

Security is an essential aspect of the system. However, there are two different levels of security. The first level concerns network security and involves authentication and encryption techniques. The second level concerns data security, meaning who can access what information and when.

\subsection{Network security}

Two things have to be secured on the network communication level:

- That the computer asking for information is indeed the one that it pretends to be

- That nobody can "listen" to the network connection and get their hands on the data being transferred

Authentication. It is envisaged to solve the first problem by an authentication system based on public and private keys, like the DSA (Digital Signature Algorithm) methods (NIST, 1999), which are included in the standard Java 1.1 platform (Campione et al., 1999).

The authentication principle is based on exchanging public key coded messages between the contacting remote communication component and the contacted one. As a result of the authentication, both components can be certain about the identity of the other one until the connection is finished.

Encryption. All confidential data sent over the network is encrypted. This could be done by the same methods as authentication. However, other solutions exist like using a connection through an SSL (Secure Socket Layer). The latest releases of standard Java tools also integrate encrypted RMI, but this has not been tested yet.

\subsection{Data security}

The first level of data security is provided by network security, in the sense that only those companies who have been declared to the system can access any 
information at all. But it is not always obvious that all companies should have access to the same information at the same time. Therefore the system provides a publishing functionality.

After a ranking has been modified for some product assortment, the user may decide to whom it will be published instantly, later or not at all. The logic component takes care of delivering the right information according to the publication restrictions for each remote request it receives.

When a new ranking is published, the logic component memorises that the companies concerned should be informed about it. This is an essential functionality, because it means that a user can ask for a list of all updated information and do the appropriate action (looking at it and adjust his own rankings accordingly or just neglect the information, for instance).

A future version of the program might include a filtering functionality, which would react automatically to certain forecasting changes made by a client or a provider. Selection criteria could be defined for deciding if a change is insignificant, interesting but not urgent or if it requires immediate attention.

\section{CONCLUSIONS}

A distributed program like the one described in this paper presents the very significant advantage of being configured and managed without the intervention of any "supervisor". This means that all sensitive data stays within the bounds of the companies involved. It also means that no member of the supply chain has more power than the others.

The biggest potential need for a "supervisor" would be for defining universal product categories for all companies. In practice, very few companies define assortments or product categories in the same way, so it is not obvious how to obtain compatible ranking information. Since the assortment forecasting method works on product rankings within one product category, it is difficult to compare the forecasts of different companies. Solutions to this problem are under way, both concerning standardisation and comparing slightly different product categories.

The concept of a distributed program should both accelerate information flow and increase forecasting precision. Forecasting changes can be immediately accessible and communicated to everyone who needs them. Forecasting precision should improve because a provider can easily get an estimation of the total sales values forecasted by all his clients and vice versa.

\section{REFERENCES}

Campione, Mary, Kathy Walrath and Alison Huml (1999). The Java Tutorial Continued, pp. 359396. Addison-Wesley, Reading, Massachusetts.
ECR Best Practices Operating Committee (1995). Category management report - enhancing consumer value in the grocery industry. Joint Industry Project on Efficient Consumer Response, USA.

Holmström, Jan (1998). Handling product range complexity: A case study on re-engineering demand forecasting. Business Process Management Journal, Volume 4 Number 3, 241-258.

NIST (National Institute of Standards and Technology) (1999). Digital Signature Standard (DSS) and Secure Hash Standard (SHS). http://csrc.nist.gov/cryptval/dss.htm

Orfali, Robert, Dan Harkey and Jeri Edwards (1997). Instant CORBA. John Wiley \& Sons, New York. 313 p.

Quelch, J. and D. Kenny (1994). Extend profits, not product lines. Harvard Business Review, September-October.

Sun Microsystems (1998). RMI Specification. http://java.sun.com/products/jdk/1.2/docs/guide/ rmi/spec/rmiTOC.doc.html 